\title{
Five-minute oscillations above granules and intergranular lanes
}

\author{
E. V. Khomenko, R. I. Kostik, and N. G. Shchukina \\ Main Astronomical Observatory, National Academy of Sciences, 03680 Kyiv-127, Ukraine
}

Received 10 May 2000 / Accepted 17 January 2001

\begin{abstract}
We discuss the links between the photospheric 5-min oscillations and the granulation pattern using a 30 -min time series of CCD spectrograms of solar granulation recorded with high spatial $\left(0^{\prime \prime} 5\right)$ and temporal (9.3 s) resolution. The observed images contain the Fe I $5324 \AA$ spectral line with good height coverage from the low photosphere up to the temperature minimum region. Amplitudes, phases and periods of the 5-min oscillations are found to be different above granules and intergranular lanes. Strong oscillations occur well separated temporally and spatially. Many features of this different behaviour can be described in the frame of a relatively simple model of wave propagation in the solar atmosphere. To that aim, we have introduced oscillations into a 3D snapshot of a theoretical time dependent solar model atmosphere. NLTE synthesis of the time series of the Fe I $5324 \AA$ line profiles was performed taking into account granular and oscillatory components of the velocity field. Both, observations and theoretical modeling, lead to similar results: (i) oscillations above granules and intergranular lanes occur with different periods; (ii) the most energetic intensity oscillations occur above intergranular lanes; the most energetic velocity oscillations occur above granules and lanes with maximum contrast, i.e. above the regions with maximum convective velocities; (iii) velocity oscillations at the lower layers of the atmosphere lead oscillations at the upper layers in intergranular lanes. In granules the phase shift is nearly zero. We conclude that differences in oscillations above granules and lanes are caused mainly by variations of the physical conditions in these structures.
\end{abstract}

Key words. Sun: photosphere-Sun: granulation - Sun: oscillations

\section{Introduction}

The spatial relation between the 5 -min oscillations and the granulation pattern has been largely debated in the literature. That discussion is important to understand the excitation mechanism of these oscillations and, hence, the internal properties of the Sun. Theoretical studies by Goldreich \& Keeley (1977), Goldreich \& Kumar (1988), Kumar \& Goldreich (1989) and Goldreich et al. (1994) suggest that acoustic waves which comprise the 5-min oscillations are stochastically generated by turbulent convection just beneath the photosphere. Moreover, Brown (1991) predicted that, since the efficiency of the acoustic wave generation significantly increases with the amplitude of the turbulent motions, most of the acoustic emission should come from rare localized events, where convective velocities are the highest. Later, Brown et al. (1992) have looked for such events using a time series of velocity variations measured in an active region near the solar disk centre. They found that, indeed, a small fraction of the volume emits a disproportionate amount of the acoustic energy.

Send offprint requests to: R. I. Kostik, e-mail: kostik@mao.kiev.ua
Goode et al. (1992) associate these localized events with expanding overshooting granules. The latter authors interpreted high spatial and temporal resolution observations of the solar velocity field made by Stebbins \& Goode (1987) as consistent with their numerical simulations. Goode et al. (1992) argued that the 5-min oscillations are excited not more than $200 \mathrm{~km}$ below the base of the photosphere. This depth is less than the value deduced by Kumar \& Lu (1991) from the higher frequency oscillation data. According to Restaino et al. (1993) the oscillatory wakes observed by these authors correspond to the events modeled by Goode et al. (1992).

A recent study of the spatial relation between the solar granulation and the 5-min oscillations by Espagnet et al. (1996) confirms the existence of strong oscillations well isolated both temporally and spatially. However, the most energetic oscillations turned out to occur only in expanding intergranular spaces with strong downflows. This conclusion disagrees with the picture in which the oscillations are excited by expanding overshooting granules. Rimmele et al. (1995) also located the acoustic events in the intergranular spaces. Observational results by Nesis et al. (1992), Solanki et al. (1996) and Nesis et al. (1997) demonstrated that enhanced turbulence in the 


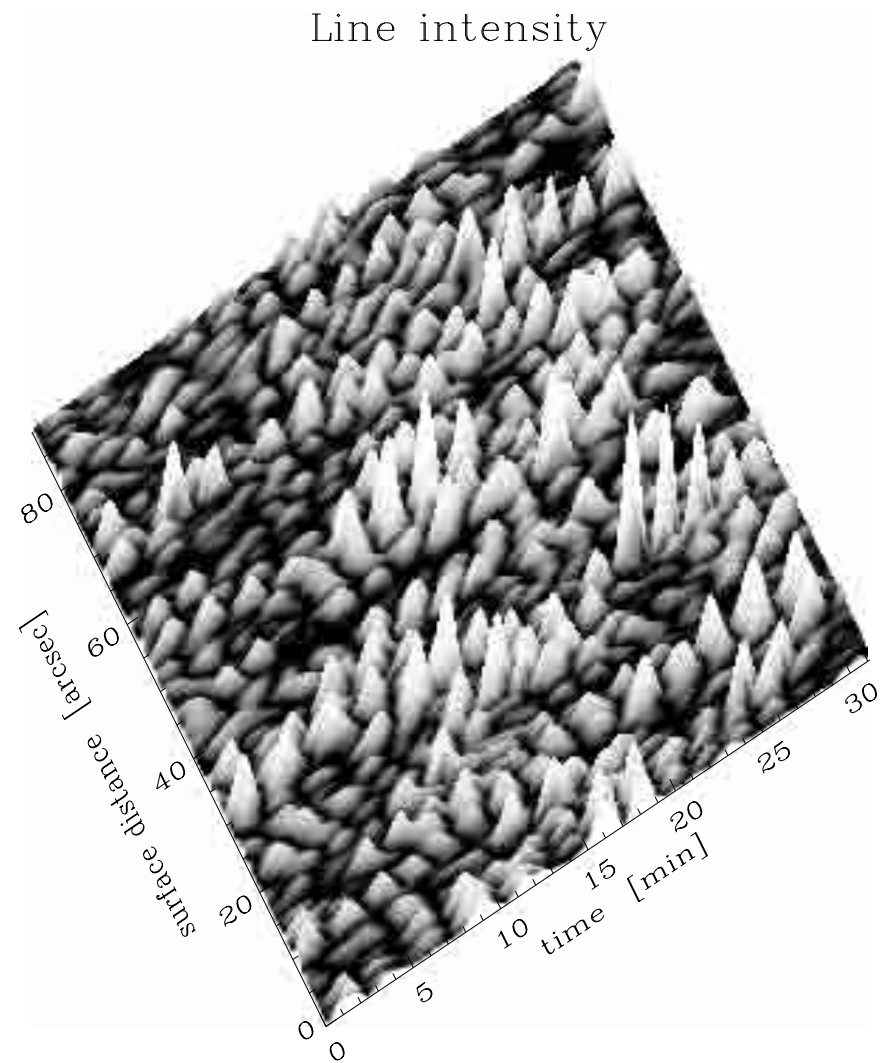

Fig. 1. Temporal evolution of the squared intensity of the oscillatory component, $\delta I_{0 \mathrm{~W}}^{2}$, on the solar surface along the spectrograph slit (ordinate)

intergranular space may be caused by strong velocity gradients at the edges of large granules.

On the other hand, analyzing $G$-band granulation filtergrams Hoekzema et al. (1998b), Hoekzema \& Rutten (1998) and Hoekzema et al. (1998a) have deduced "that the photospheric $5-$ min oscillations are primarily global and rather insensitive to the local fine structure".

We conclude that, despite considerable efforts by different researchers, the issue of the links between the 5-min oscillations and the granulation remains open. In this paper we tackle this problem using high (spatial and temporal) resolution observations of the spectral line Fe I $5324 \AA$. We complement the analysis of observations with theoretical modeling and show that the differences in oscillations above granules and intergranular lanes are caused mainly by the differences in physical conditions in these structures.

\section{Observations}

The observations were carried out in August 1996 at the $70 \mathrm{~cm}$ German Vacuum Tower Telescope of the Observatorio del Teide, Tenerife. The telescope and spectrograph are described by Schröter et al. (1985). The CCD spectrograms contain the photospheric line Fe I $5324 \AA$. The characteristics of the line are given in Table 1 . Its columns specify the wavelength $\lambda$, the excitation potential of the lower level EPL, the observed central line depth $D$,

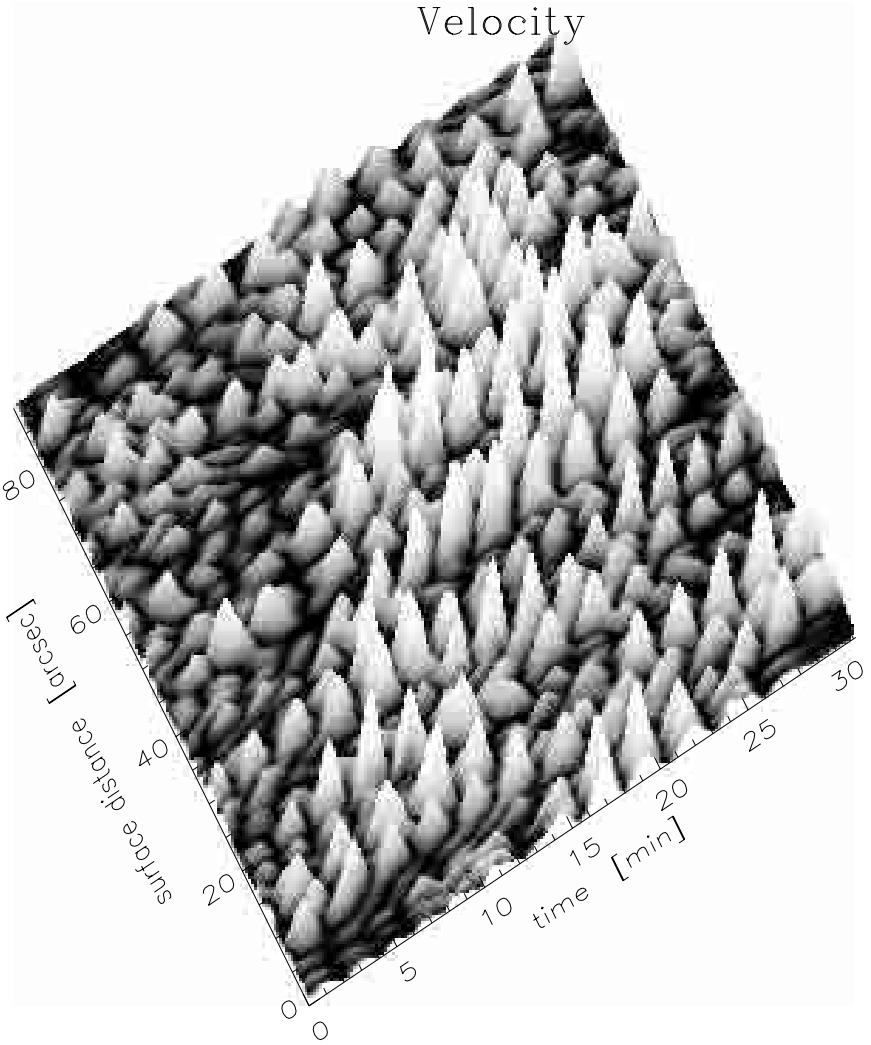

Fig. 2. Same as Fig. 1, but for the squared velocity of the oscillatory component, $\delta V_{0 \mathrm{~W}}^{2}$

the equivalent width $W$ (the latter two values are averaged over space and time) and the line core formation height $H$. The wavelength and EPL values were taken from Moore et al. (1966). The $D$ and $W$ values were measured following Gurtovenko \& Kostik (1989). The height $H$ for the Fe I line was obtained from the NLTE $1.5 \mathrm{D}$ calculations performed by us using the solar convection simulations by Stein \& Nordlund (1998) and Asplund et al. (2000) as $3 \mathrm{D}$ model atmosphere. The $H$ value is model dependent and on average is around $500 \mathrm{~km}$. The oscillator strength $\log g f$ is taken from Holweger et al. (1991).

The line core intensity is formed rather high in the photosphere where significant variations of brightness and velocity caused by wave motions are expected. The line formation height region extends from the bottom of the photosphere to the temperature minimum which gives an opportunity to study the height dependence of oscillatory properties.

The observed area was near the quiet solar disk centre. Magnetically active regions were avoided by controlling the entrance slit position using $\mathrm{H} \alpha$ and Ca II K slit-jaw images. We used a CCD camera with $1024 \times 1024$ pixels,

Table 1. Parameters of the line observed

\begin{tabular}{cccccrc}
\hline $\begin{array}{c}\lambda \\
{[\AA]}\end{array}$ & Elem. & $\begin{array}{c}\text { EPL } \\
{[\mathrm{eV}]}\end{array}$ & $\log g f$ & $D$ & $\begin{array}{r}W \\
{[\mathrm{~m} \AA]}\end{array}$ & $\begin{array}{c}H \\
{[\mathrm{~km}]}\end{array}$ \\
\hline 5324.185 & Fe I & 3.21 & -0.10 & 0.861 & 318 & 500 \\
\hline
\end{tabular}




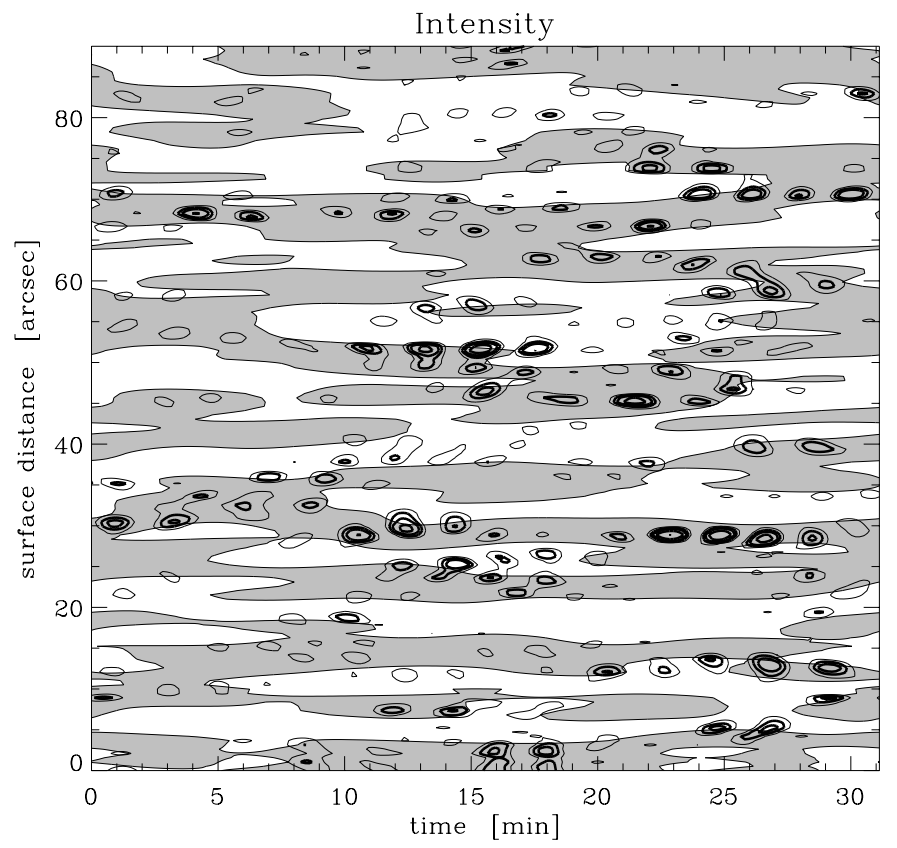

Fig. 3. Temporal evolution of the continuum contrast of the convective component, $\delta I_{\mathrm{c}}$, along the slit shown simultaneously with the temporal variation of the squared intensity of the oscillatory component, $\delta I_{0 \mathrm{~W}}^{2}$. Light areas are granules, dark areas are intergranular lanes. The $\delta I_{0 \mathrm{~W}}^{2}$ isolines are $0.1,0.2$, $0.3,0.5$, and 1.0 in units of their maximum values

in a $2 \times 2$ binning mode. A pixel size in this mode was 0.174 arcsec in the spatial direction along the slit. The dispersion of the spectra was about $0.10 \AA / \mathrm{mm}$ at $6000 \AA$. The entrance slit width of the spectrograph was set to $80 \mu \mathrm{m}$, i.e. 0.38 arcsec on the solar disk. Thus, each overall image corresponds to an area on the solar surface about $0.38 \times 89 \operatorname{arcsec}^{2}$. The Fe I 5324 line spectrograms were taken every $9.3 \mathrm{~s}$ with an integration time of $5.7 \mathrm{~s}$. The time series covered about 31 minutes and included 200 images in total.

\section{Data reduction}

The reduction of CCD images followed the standard procedure. After dark current subtraction and flat fielding all spectral frames were aligned to have the dispersion exactly parallel to the pixel rows. All the frames were normalized to their average continuum intensity to remove the fluctuations of the sky transparency. Since the actual (seeing-limited) spatial resolution of our observations was not better than 0.5 arcsec, three successive pixel rows were averaged in the spatial direction along the slit.

For every averaged spectral pixel row $i(i=1-170)$ of every image $j(j=1-200)$ we inferred intensity and velocity signals at 11 levels of the line profile $\delta I_{1}(i, j)$, $(l=0-10)$ and $\delta V_{l}(i, j),(l=0-10)$, where $l=0$ corresponds to the line core. The procedure was performed applying the method described by Stebbins \& Goode (1987). The presence of a wave must disturb both the position and shape of a line profile. For that reason, velocity was

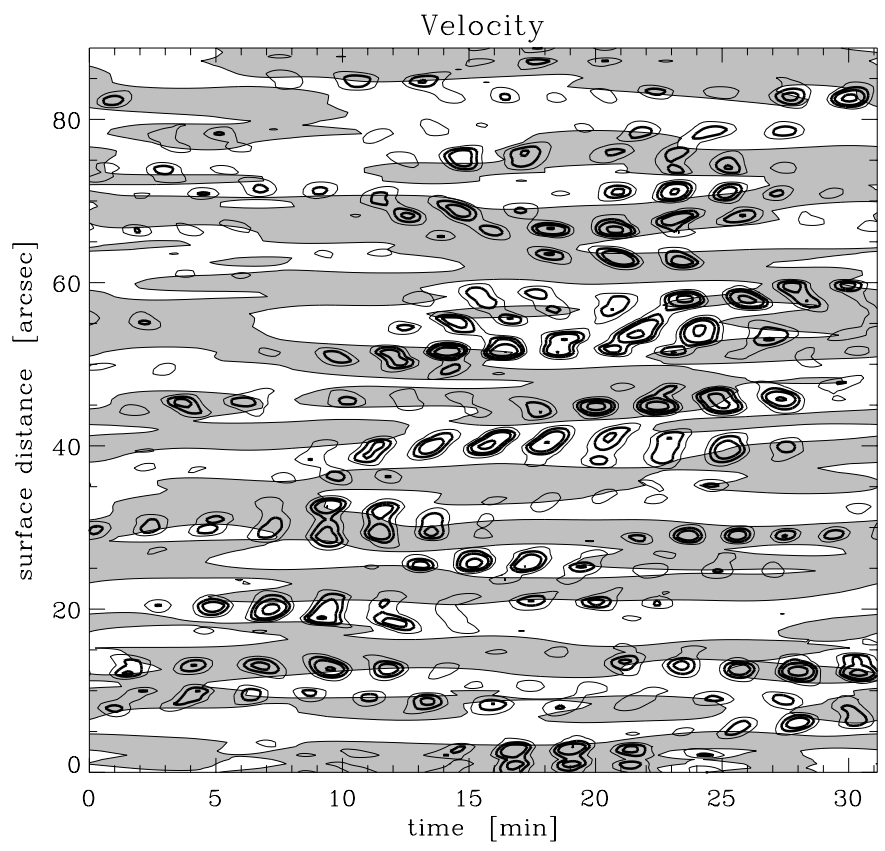

Fig. 4. Same as in Fig. 3 but for the square of the velocity oscillatory component, $\delta V_{0 \mathrm{~W}}^{2}$

defined as the midpoint of that section of the profile having a certain spectral width. The intensity was simply the level of that section. These 11 line intensities of course do not reflect 11 independent atmospheric layers. But we may expect that velocities and intensities from the progressively higher line sections correspond to progressively deeper layers of the atmosphere.

Another line parameter under study was the continuum intensity at every spectral row of every image $I_{\mathrm{c}}(i, j)$. Since the CCD images covered only about $2 \AA$ of the spectrum the true line continuum was not available for measurements. So we used a nearby local continuum. For the Fe I 5324 line it was placed at a distance of, approximately, $1.1 \AA$ from the line centre. According to the Liège atlas (Delbouille et al. 1973) the residual intensity in the spatially averaged solar spectrum reaches there $\sim 97 \%$ (continuum level $=100 \%$ ). For each single spectrum row the $I_{\mathrm{c}}$ value was the average over $10-20$ pixels.

The variations of all parameters were taken with respect to those of the temporal and spatial average spectrum. Since our study deals only with the relative changes of the line parameters no correction for the influence of the spectrograph instrumental profile and scattered light has been made.

The variations in the line parameters under study are induced mainly by convective and wave motions. In order to separate granular and oscillatory components of the velocity field, we constructed a diagnostic $k-\omega$ diagram. Based on that diagram, we restricted the wave motions by temporal frequency range $\omega=1.8-5.7 \mathrm{mHz}$ $(T=170-560 \mathrm{~s})$ and spatial frequency lower than $0.8 \mathrm{Mm}^{-1}$. The latter means that all the structures of size below 1.6 arcsec were excluded. Convective motions 

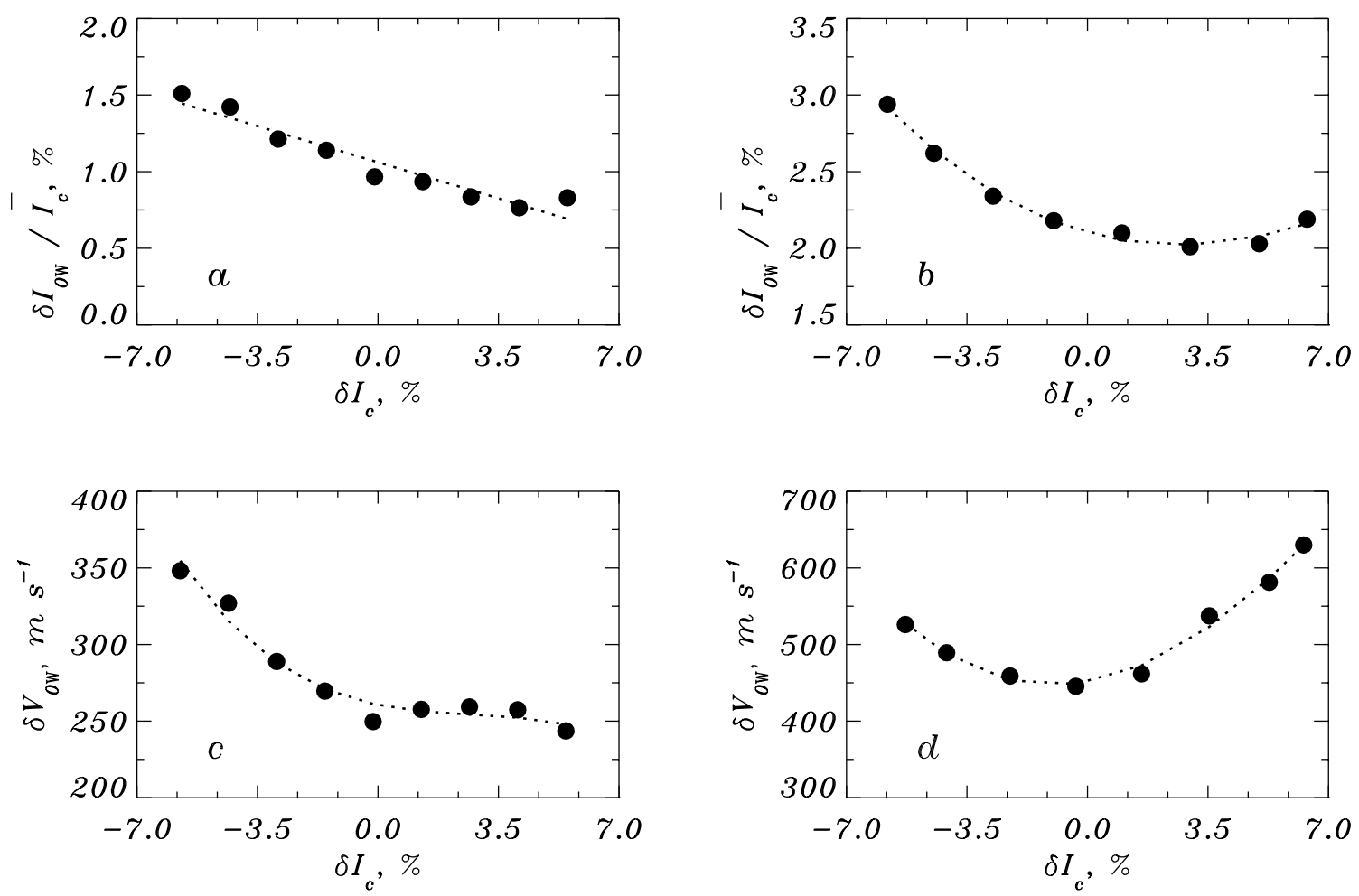

Fig. 5. a) and b) Amplitudes of the line core intensity oscillations $\delta I_{0 \mathrm{~W}} / \bar{I}_{\mathrm{c}}$ as a function of continuum contrast $\delta I_{\mathrm{c}}=\left(I_{\mathrm{c}}-\bar{I}_{\mathrm{c}}\right) / \bar{I}_{\mathrm{c}}$. c) and $\mathbf{d}$ ) the same but for velocity oscillations $\delta V_{0 \mathrm{w}}$. Amplitudes in panels a) and c) are measured as extreme values in each oscillating element and then averaged over contrast intervals. Amplitudes in panels b) and d) are measured as maximum values in these elements

were limited only in the temporal frequency $\omega<2.2 \mathrm{mHz}$ $(T>450 \mathrm{~s})$. To this end we applied the corresponding low- and high-pass filters. The oscillatory and convective intensity components were separated in a similar way.

\section{Results and discussion}

\subsection{Amplitudes of velocity and intensity oscillations}

Figures 1-4 show the temporal evolution of the acoustic events observed in the granular pattern for 170 elements along the spectrograph slit. Each element corresponds to an area of $0.38 \times 0.52 \operatorname{arcsec}^{2}$. Acoustic events are represented by the squared amplitudes of the wave component corresponding to the line core intensity (Figs. 13 ) and velocity (Figs. 2-4) variations $\left(\delta I_{0 \mathrm{~W}}^{2}\right.$ and $\delta V_{0 \mathrm{~W}}^{2}$, respectively).

Granules and intergranules were defined according to their continuum intensity. The points with continuum intensity larger than the average were taken as granules (and the opposite for intergranules). Granules were found to occupy only $47.8 \%$ of the total area of the observed region which is in a good agreement with recent results (47-48\%) of Hirzberger et al. (1997) and Hoekzema \& Rutten (1998). Note also that the mean contrast $\delta \bar{I}_{\mathrm{c}}$ for granules and intergranular lanes were $+2.1 \%$ and $-2.1 \%$, respectively.

A wave pattern of intensity and velocity fluctuations is clearly visible in Figs. 1-4, with a period of about $5 \mathrm{~min}$.
Wave trains last only a few periods. Strong oscillations are well separated temporally and spatially. It is noticeable that the distribution of the velocity and intensity oscillatory cells over the surface is different. There are regions where velocity oscillations are enhanced while intensity oscillations are weakened. It is clear that intense 5-min intensity oscillations avoid bright areas (granules) and tend to appear above dark intergranular lanes or at the boundaries of granules. Strong velocity oscillations seem to be more insensitive to the local fine structure: they are observed above granules as well as above dark lanes.

To gain more information about the amplitude distribution with continuum contrast, we measured the wave components of the velocity and intensity variations $\delta V_{0 \mathrm{~W}}$ and $\delta I_{0 \mathrm{~W}}$ at the moments when they reached their maximum values. The continuum contrast $\delta I_{\mathrm{c}}$ was also determined at these moments. The range of amplitudes for a given value of contrast turned out to be quite large. Notwithstanding, we splitted the contrast range into several equally sized bins and averaged the oscillatory amplitudes corresponding to each bin. The averaged amplitudes show a clear dependence on granulation contrast. Figures 5a and c demonstrate this result. The intensity amplitude grows linearly by almost a factor of two as the contrast changes from $5.5 \%$ to $-5.5 \%$. The largest $\delta I_{0 \mathrm{~W}}$ amplitudes are observed above intergranular lanes, i.e. they are the larger the darker the lanes. The velocity amplitude depends slightly on the granulation contrast 
and increases only by a factor of 1.4 over the same interval of continuum contrast. The amplitude $\delta V_{0 \mathrm{~W}}$ is nearly constant above granules with different contrast and increases rapidly above intergranular lanes as they become darker. So, in general the large-amplitude oscillations are suppressed above granules.

This conclusion changes markedly if we consider not all the amplitudes but only the maximum amplitudes in the oscillating element. Figures $5 \mathrm{~b}$ and $\mathrm{d}$ show the relationships for this case. The velocity amplitudes are now greater for the higher granulation contrasts, showing that the velocity oscillations are particulary strong above the brightest granules. Intensity oscillatory amplitudes at the wave train maximum remain following the same trend as before, namely, the strongest oscillations occur above intergranular lanes only. We conclude that the most powerful intensity oscillations occur predominantly above the dark intergranular lanes, while there is no such tendency for velocity oscillations. These results disagree with those by Espagnet et al. (1996), who argue that the most energetic velocity oscillations occur only above intergranular lanes. It is particulary interesting taking into account that the formation height of the core intensity of the $\mathrm{Na}_{\mathrm{I}} \mathrm{D}_{2}$ line $(H=500 \mathrm{~km})$, observed by Espagnet et al. (1996) is close to the formation height of the Fe I $5324 \AA$ line used in our study (see Table 1). One possible reason is that the duration of the spectroscopic observations by these authors is a factor of 2 shorter $(16 \mathrm{~min})$ than ours.

\subsection{Long-living granules}

One of the ways to investigate the links between 5-min oscillations and granulation is to isolate an individual granule and intergranular lane and then observe the evolution of oscillations above them. However, there is a problem related to the short lifetime of the granulation pattern. The granular mean lifetime is $\sim 10 \mathrm{~min}$ (Alissandrakis et al. 1987; Title et al. 1989; Hirzberger et al. 1999). As a consequence, we can not apply the same methods of spectral analysis to such a short time series (2 periods of 5 -min oscillations). There are some possible solutions of this problem. One way is to construct an "artificial granule", a method firstly applied by Kostik \& Shchukina (1999). The spectrograms are averaged along the slit positions for all continuum intensities above/below a certain threshold. The temporal evolution of the average spectra is then constructed and its oscillatory behaviour analysed. The advantages and shortcoming of this method are discussed by Kostik \& Shchukina (1999). Another way (the one we follow in this work) is to select long-living granules and analyse the oscillation above them. We are doing that in this study. We found $23 \%$ of granules (and intergranular lanes) in our observations with a lifetime longer than $20 \mathrm{~min}$. This value does not contradict the results of the granulation study by Title et al. (1989) and Hirzberger et al. (1999).
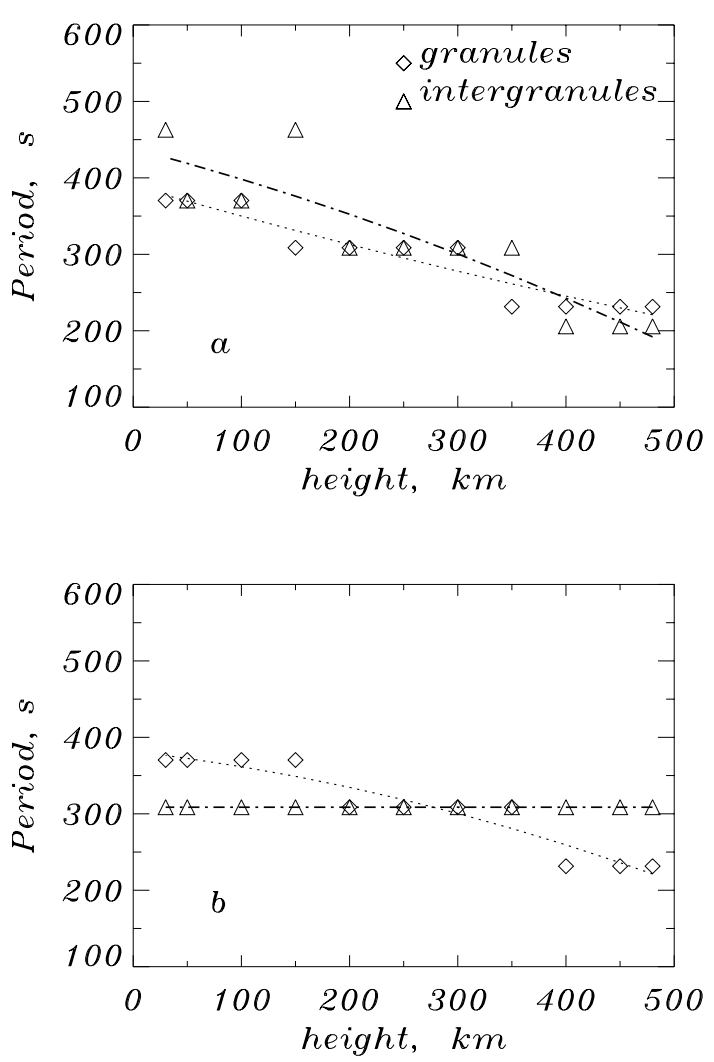

Fig. 6. a) Period of the most powerful intensity oscillations above granules and intergranular lanes as a function of height. b) the same as a) but for velocity oscillations. The dotted and dashed-dotted lines represent the trends of the variations

The periods at which the maximum power is observed for this long-living granules are displayed in Fig. 6. The matter above granules exhibits velocity oscillations with larger periods in the deeper layers and with lower periods in the higher layers. Contrarily, there is no such dependence for oscillations above the intergranular lanes, where the period is constant through the photosphere. In contrast, the intensity oscillation periods are not different for granules and intergranular lanes.

Unfortunately, we can not obtain a higher frequency resolution, since it is determined by the maximum lifetime of the granulation. The results shown in Fig. 6 should be considered as preliminary. Anyway, they represent a clear hint that velocity oscillations appear above granules and intergranular lanes with different periods. The difference in periods can possibly be explained by the Doppler shift caused by convective velocities in granules and intergranular lanes (see Sect. 4.3).

\subsection{Simulation of wave propagation in the photosphere}

It seems natural that oscillations above granules and intergranular lanes are not identical because the physical conditions in these regions vary substantially. Even under the assumption that oscillations at the bottom are generated with equal amplitudes dissimilarities should occur at 
the top of the photosphere if waves propagate through considerably different media such as granules and intergranular lanes. Firstly, the temperature variations in granules and lanes produce changes in sound speed and radiative relaxation time and also modify the critical frequency under which oscillations become evanescent. Secondly, differences in pressure and density affect the scale height to which an oscillatory amplitude is sensitive. And last but not least, oscillations propagate in a moving medium with upflows, downflows and horizontal flows. Thus, the measured oscillatory frequencies are shifted by Doppler effect.

\subsubsection{Model of the 5-min oscillations}

On the grounds stated above, we found it reasonable to reconsider from a theoretical point of view the wave propagation in the solar atmosphere and to pay more attention to the effect of oscillations on a line profile. The main difference between our work and similar studies of other authors (e.g. Marmolino \& Stebbins 1989; Bertello \& Caccin 1990; Marmolino \& Severino 1991, and references therein), is that we considered wave propagation in a moving medium. In the cited papers, the granulation component of the velocity field was neglected and only oscillatory perturbations were considered. In the present study we make an attempt to include both velocity components.

A comprehensive analysis of the propagation of the acoustic-gravity waves in a radiating fluid is given by Mihalas \& Mihalas (1984). We follow this analysis and the scheme given by Noyes \& Leighton (1963) and then developed by Kostik (1974) and Schmieder (1977). We solved analytically the hydrodynamical equations of mass, momentum and energy conservation:

$\rho \frac{\mathrm{d} \boldsymbol{V}}{\mathrm{d} t}=-\nabla P+\boldsymbol{g} \rho$

$\frac{\partial \rho}{\partial t}=-\nabla(\rho \boldsymbol{V})$

$\frac{\mathrm{d} Q}{\mathrm{~d} t}=c_{\mathrm{v}} \frac{\mathrm{d} T}{\mathrm{~d} t}-\frac{R T}{\rho} \frac{\mathrm{d} \rho}{\mathrm{d} t}$

where $\mathrm{d} / \mathrm{d} t=\partial / \partial t+\nabla \boldsymbol{V}$ is the total derivative, $\mathrm{d} Q / \mathrm{d} t$ is the net rate of radiative energy input (per unit volume) to the gas from external sources; other notations are standard. We assumed that there is no magnetic field; that viscosity and thermal conduction terms can be omitted; that the effects of solar rotation are negligible; and, that to the 0th order, the atmosphere is plane parallel, horizontally homogeneous and in radiative equilibrium. Matter is described by a suitable equation of state (no effects of ionization are considered):

$\frac{T_{1}}{T_{0}}=\frac{P_{1}}{P_{0}}-\frac{\rho_{1}}{\rho_{0}}$.

The effects of radiative relaxation, the main source of dissipation for linear waves in the solar photosphere, is taken into account by using the Newtonian cooling approximation.
The motion is restricted to one dimension. Hence, we consider vertically propagating acoustic-gravity waves. Internal and surface gravity waves are excluded. The solution of the system of partial differential equations was obtained by approximating the atmosphere as a series of isothermal layers each of a given thickness $(10 \mathrm{~km})$ moving with a constant velocity. In each layer we linearized the fundamental equations with:

$T=T_{0}+T_{1}, P=P_{0}+P_{1}, \rho=\rho_{0}+\rho_{1}, V=V_{0}+V_{1}$.

The dimensionless and linearized equations then become (to the 1st order):

$$
\begin{aligned}
& \frac{\partial W_{1}}{\partial t}+W_{0} \frac{\partial W_{1}}{\partial z}=-\frac{\partial}{\partial z}(R+\Theta)+2 \Theta \\
& \frac{\partial R}{\partial t}+W_{0} \frac{\partial R}{\partial z}=2 W_{0} R-\frac{\partial W_{1}}{\partial z}+2 W_{1} \\
& \frac{\partial \Theta}{\partial t}+W_{0} \frac{\partial \Theta}{\partial z}=-\frac{1}{\tau_{R R}} \Theta-(\gamma-1) \frac{\partial W_{1}}{\partial z}
\end{aligned}
$$

Here, the vertical velocity $W$ is measured in units of the sound speed $\left(W_{0,1}=V_{0,1} / C_{\mathrm{s}}\right), \Theta=T_{1} / T_{0}$ and $R=\rho_{1} / \rho_{0}$ are in units of the corresponding unperturbed values, and the distance $z=\tilde{z} / 2 H$ is in units of the doubled pressure scale height $H^{-1}=-\nabla P / P$ at the given layer. The system has an exponential solution at a fixed frequency $\omega$ of the form:

$W_{1}, \Theta, R \sim \mathrm{e}^{z\left(1-k_{i}\right)} \mathrm{e}^{-i k_{\mathrm{r}}\left(t V_{\mathrm{ph}}-t W_{0}-z\right)}$

where $V_{\mathrm{ph}}=\omega / k_{\mathrm{r}}$ is the phase velocity of the wave, $k_{\mathrm{r}}$ and $k_{\mathrm{i}}$ are the real and imaginary parts of the vertical wave number $k_{z}$. Only upward modes can be excited in an isothermal atmosphere perturbed from below. Depending on the frequency these modes can either propagate or become evanescent if the condition $\omega<\omega_{\mathrm{c}}$ satisfies (where $\omega_{\mathrm{c}}$ stands for a critical frequency). Therefore, by selecting an upward solution of Eqs. (6-8) the vertical velocity can be written as follows:

$W_{1}=V_{0 \mathrm{w}} \mathrm{e}^{z\left(1-k_{\mathrm{i}}\right)} \sin \left(\left(\omega-W_{0} k_{\mathrm{r}}\right) t-k_{\mathrm{r}} z\right)$.

The corresponding waveform for the thermodynamic perturbations $T_{1} / T_{0}$ and $\rho_{1} / \rho_{0}$ were obtained from the polarization relations, and the pressure perturbations were found from the equation of state (Eq. (4)):

$$
\begin{aligned}
& \frac{T_{1}}{T_{0}}=|\Theta| \mathrm{e}^{z\left(1-k_{\mathrm{i}}\right)} \sin \left(\left(\omega-W_{0} k_{\mathrm{r}}\right) t-k_{\mathrm{r}} z+\phi_{T V}\right) \\
& \frac{\rho_{1}}{\rho_{0}}=|R| \mathrm{e}^{z\left(1-k_{\mathrm{i}}\right)} \sin \left(\left(\omega-W_{0} k_{\mathrm{r}}\right) t-k_{\mathrm{r}} z+\phi_{\rho V}\right) .
\end{aligned}
$$

Positive direction of the vertical axis $z$ in our study was chosen downward into the Sun, and, thus, waves moving toward the observer have negative velocity. Variations of the thermodynamical quantities (Eqs. (10)-(12)) were introduced in a 3D model atmosphere and then profiles of the Fe I $5324 \AA$ line were synthesized. 


\subsubsection{Model atmosphere and profile synthesis}

The 3D model atmosphere used in our study is a snapshot of the most recent numerical simulations by Asplund et al. (2000). The model has been obtained with a 3D, timedependent, compressible, radiative hydrodynamic code developed to study solar and stellar surface convection (e.g. Stein \& Nordlund 1989; Nordlund \& Stein 1990; Asplund et al. 1999). The numerical details involved in the 3D computations are described by Stein \& Nordlund (1998). We interpolated the original simulations of size $200 \times 200 \times 82$ gridpoints which has physical dimensions of $6.0 \times 6.0 \times 3.8 \mathrm{Mm}^{3}$ with nearly $1.0 \mathrm{Mm}$ being located above the continuum optical depth unity. The interpolated cube has $50 \times 50 \times 82$ gridpoints. The simulated box includes about 15 granules. We considered the 3D snapshot as a set of 1D granular/intergranular model atmospheres. For the line profiles calculations we randomly chose a slice from the snapshot which contains 5 granules separated by intergranular lanes. The average temperature difference between them is about $1000 \mathrm{~K}$ at the height $H=0 \mathrm{~km}$. We extracted a set of $501 \mathrm{D}$ granular and intergranular model atmospheres by taking the height variation of the thermodynamical quantities at selected gridpoints of the slice. We interpolated them to a finer grid with 102 height points each $10 \mathrm{~km}$ apart.

For each model we computed the line profile by solving the radiative transfer equation for a moving atmosphere perturbed by oscillations. The NLTE study of Fe I line formation in the solar atmosphere by Shchukina \& Trujillo Bueno $(1997,2000)$ shows that NLTE effects are potentially important for spatially and temporally resolved profiles of the Fe I $5324 \AA$ line. To this end, we used as input in the formal radiative transfer solution NLTE departure coefficients found from the self-consistent solution of the kinetic and radiative transfer equations for the realistic iron atomic model and unperturbed granulation models. We assumed that the perturbations caused by waves in the atmosphere are small and do not change the departure coefficients of the perturbed model atmosphere. The details of our NLTE computations are described by Shchukina \& Trujillo Bueno (2000). Here let it suffice to mention that we used powerful iterative multilevel transfer methods and formal solvers that are capable of handling efficiently hundred of transitions (see Auer et al. 1994; Trujillo Bueno \& Fabiani Bendicho 1995). Our atomic model gives account of multiplet structure and includes over 250 levels and nearly $500 \mathrm{UV}$, optical and IR transitions.

Time series of 40 profiles with the real time step of $9.3 \mathrm{~s}$, close to the observed value, were synthesized in each of the 50 models. The overall time of computations was 1 wave period $(\sim 5 \mathrm{~min})$. The initial amplitude of the wave perturbation was chosen equal $V_{0}=100 \mathrm{~m} \mathrm{~s}^{-1}$, and the initial period was $268 \mathrm{~s}$. The value of $V_{0}$ was taken from our observations of the spatially averaged oscillations at height $H=0 \mathrm{~km}$. The phases of oscillatons were taken equal at the bottom level in all the models. Then we solved

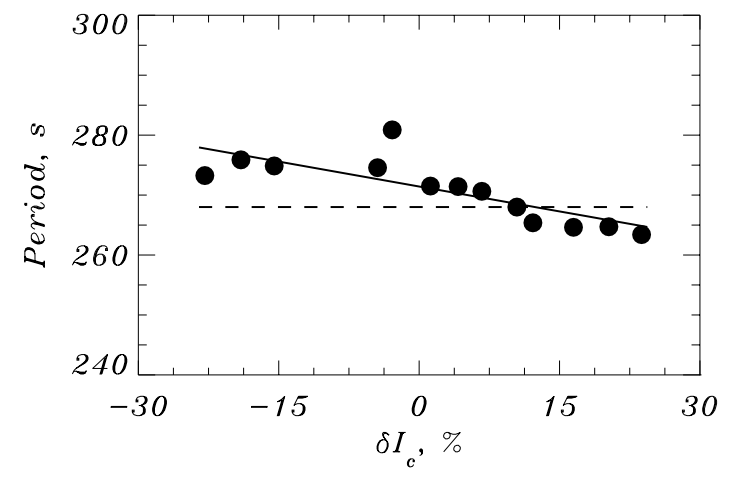

Fig. 7. Periods of the simulated oscillations as a function of the continuum contrast $\delta I_{\mathrm{c}}$. The initial period for the oscillations was chosen equal $268 \mathrm{~s}$ (dashed line). Wave propagates upward. Upflows in granules produce shifts in the visible periods toward smaller periods and downflows in intergranular lanes toward larger periods. Dots represent the oscillation periods at the height of the Fe I $5324 \AA$ line core formation

the inverse problem and calculated the intensity and velocity oscillations at 11 levels of the computed line profiles applying the same method as for the observational data (Sect. 3).

In our study we use only one slice in a single snapshot of the 3D model. We realize that in order to investigate properly the links between 5-min oscillations and granulation it would be desirable to simulate oscillations in the time sequence of snapshots. This goal can be achieved at the enormous numerical expense caused by multilevel radiative transfer solution in $3 \mathrm{D}$ dynamic media. Prior to performing (and understanding) such computations we feel it important to first investigate carefully the oscillatory parameter variations neglecting time evolution of the atmospheric parameters of granules/lanes. We would like to stress that since the mean granular lifetime is about $10 \mathrm{~min}$ the variations in granulation pattern during 5 -min wave period will be hardly crucial.

The results of our simulations in comparison with observations are shown in Figs. 7-10.

\subsection{Periods}

The presence of non-zero vertical velocity fields leads to Doppler shifts in the oscillatory frequencies. The effective frequency which we can measure is not constant now, but is modified by velocity:

$\tilde{\omega}=\omega-W_{0} k_{\mathrm{r}}$.

Figure 7 shows the shifts in periods derived from the above formula as a function of continuum contrast. Granules have mainly negative velocities (upward motion, $W_{0}<0$ ), and lanes mainly positive ones (downward motion, $W_{0}>$ 0 ). Therefore the effective period $\tilde{\omega}$ of the upward propagating wave is smaller in granules and larger in intergranular lanes (Fig. 7). For the downward propagating wave the situation will be vice versa: we should observe an increase of the period above granules. The convective velocities in 

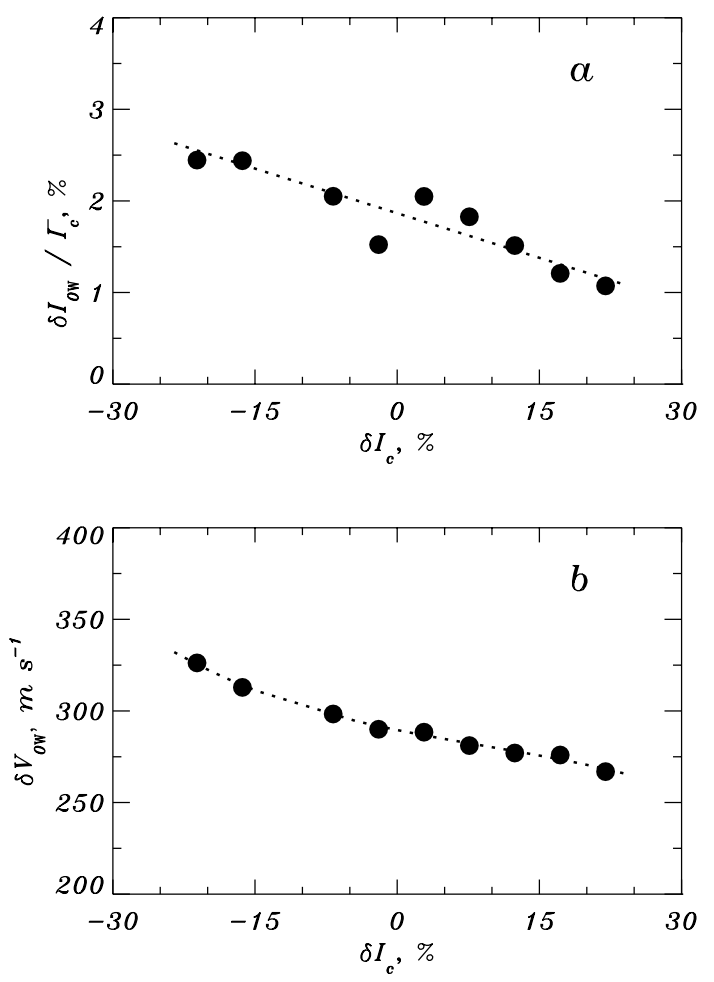

Fig. 8. a) Amplitudes of the intensity oscillations as a function of continuum contrast for the calculated Fe I $5324 \AA$ line profiles. b) the same as a) but for velocity oscillations

the 3D model lead to the maximum period splitting of $15 \mathrm{~s}$ at a height of $500 \mathrm{~km}$. This is much less than the difference seen in the observations (Fig. 6). But we are not sure whether this difference is caused by the shortness of the time series and, hence, not sufficient frequency resolution or by the high convective velocities. According to Asplund et al. (2000) the 3D model atmosphere used in our study describes granulation rather well, so there is no reason to increase velocities. The period is lower above granules which means that the observed waves propagate upward. This is in agreement with the results of the oscillatory phases examination performed in Sect. 4.6.

\subsection{Theoretical amplitudes}

Figures $8 \mathrm{a}$ and $\mathrm{b}$ display the dependence of the simulated line center intensity $\delta I_{0 \mathrm{~W}}$ and velocity $\delta V_{0 \mathrm{~W}}$ amplitudes on continuum contrast. It is noticeable that the range of the continuum contrast $\delta I_{\mathrm{c}}$ of the profiles emergent from the $3 \mathrm{D}$ model atmosphere is nearly 4 times larger than the observed contrast range (panels a and c). The theoretical profiles have better spatial resolution $(125 \mathrm{~km}$ in comparison with no more than $500 \mathrm{~km}$ in observations) and are not affected by seeing of the atmosphere and instrumental profile of the telescope. Outstanding stratospheric observations of solar granulation by Karpinsky (1990) with a spatial resolution of $0.22(\approx 200 \mathrm{~km})$ reveal values of $\delta I_{\mathrm{c}}$ as large as $22 \%$. This contrast is compatible with that shown in the Fig. 8. To reduce a continuum contrast in the hydrodynamical simulations a convolution of the profiles with a suitable point-spread-function is usually applied. This procedure allows to decrease the continuum contrast to about $8 \%$, which is more or less compatible with observations (Asplund et al. 2000). We did not perform any convolution but, instead of that, we assumed the computed contrast range to be equivalent to the observed one, giving us the possibility to compare observational and theoretical results in Figs. 5 and 8.

The amplitudes of the computed intensity oscillations happen to be two times larger that the observed intensity amplitudes which we think should be also attributed to the better spatial resolution. However, as it follows from comparison of the Figs. $5 \mathrm{c}$ and 8b, the observed and calculated velocity amplitudes agree rather well. We compared the inclination of the curves in Figs. 5 and 8 assuming that the observed and simulated continuum contrasts correspond to each other. The intensity amplitude grows by a factor of 2.3 and velocity by a factor of 1.3 as we pass from the brightest granules to the darkest lanes. Taking into account that the observations give factors 2.1 and 1.4 respectively, we found the agreement not to be bad for the simplified theory used.

Thus, we conclude that even if all initial and boundary conditions are equal at $H=0$, oscillations above granules and intergranular lanes turn out to be different at heights of about $500 \mathrm{~km}$, where the core of our Fe I line is formed. Since we have not applied any hypothesis concerning wave excitation, the changes may be ascribed only to the differences in the physical structure of granules and lanes.

\subsection{Height dependence of the amplitudes and phases}

The difference between oscillations above granules and intergranular lanes is present not only at heights around $500 \mathrm{~km}$ but at all layers of the photosphere. Figure 9 displays empirical and theoretical runs of the intensity and velocity oscillatory amplitudes with height. Following Sect. 4.1 we measured amplitudes of oscillations and continuum contrast at the moments when the former reached the maximum and minimum values. This was done for oscillations at 11 levels of both the observed and the synthesized line profiles (see Sect. 3). We splitted amplitudes into two granular and intergranular classes $\left(\delta I_{\mathrm{c}}(i, j)>0\right.$ and $\left.\delta I_{\mathrm{c}}(i, j)<0\right)$ and averaged them within the same class at the given level. Oscillations at each level of the profile were ascribed to a certain height in the photosphere. Since we did not aim at extract an exact height information but rather tried to make an estimation and to better visualize the data we did not calculate response functions to derive precise heights of formation of these points. Instead, we employed heights where the optical depth at the given wavelength was equal to unity $\left(\tau_{\nu}=1\right)$. To calculate the depth, two average model atmospheres of granule and intergranular lane were utilized from the horizontal cut of the 3D snapshot. 

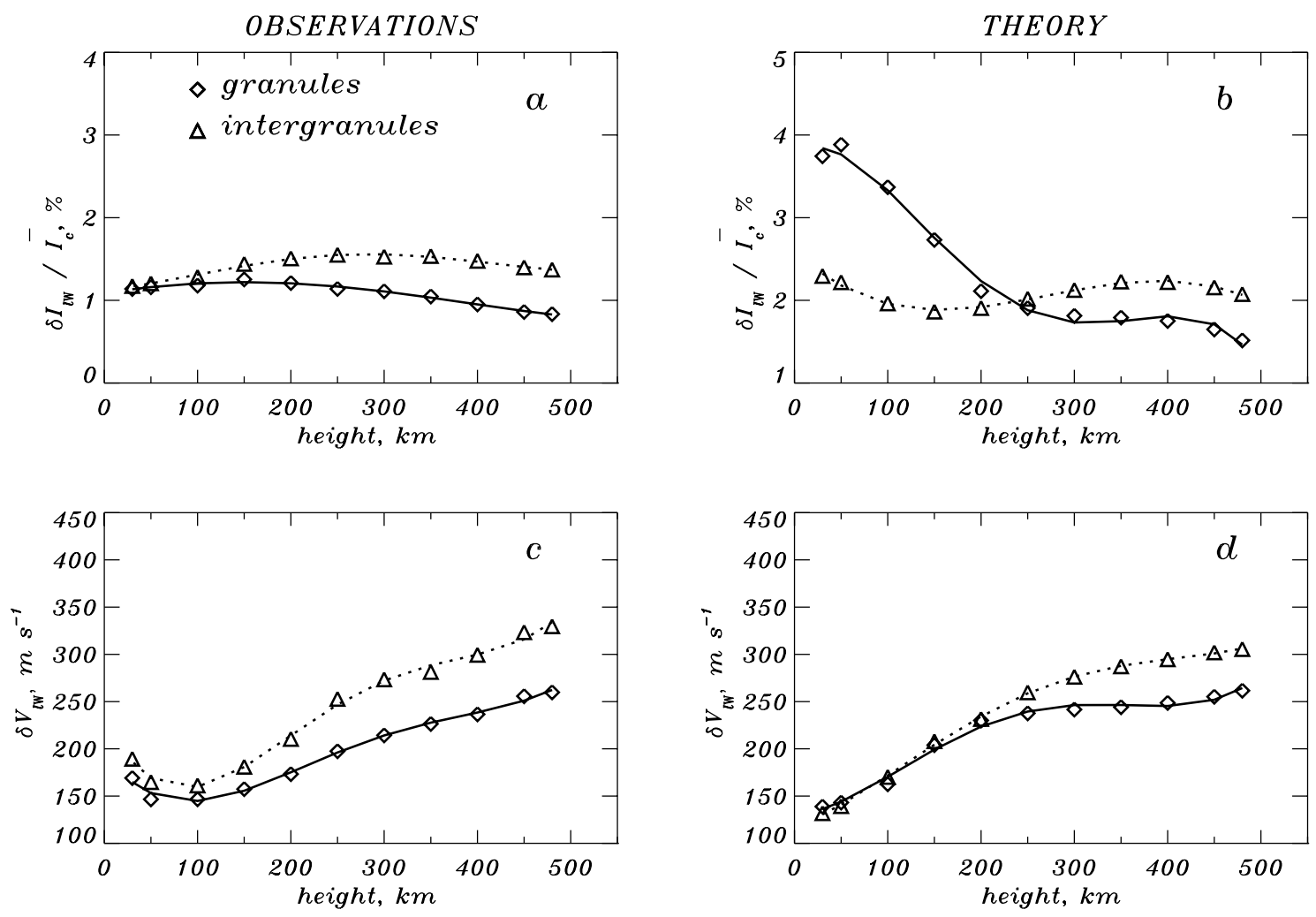

Fig. 9. a) and b) Height dependence of the intensity oscillatory amplitude $\delta I_{\mathrm{lW}} / \bar{I}_{\mathrm{c}}$ above granules and intergranular lanes. c) and $\mathbf{d}$ ) the same but for velocity oscillatory amplitude. Amplitudes were measured at different heights and then averaged separately for oscillations above granules and lanes

Oscillations above intergranular lanes turn out to have larger amplitudes than above granules throughout the photosphere both for velocity and intensity. The difference between the amplitudes grows with height. Empirical and theoretical intensity amplitudes agree only in the layers above $200 \mathrm{~km}$. In deeper layers theory predicts a rapid enhancement of the amplitude and a larger value above granules. The theoretical intensity amplitude run resembles in high degree the temperature run in granule and intergranular lane of the 3D model. This is not surprising since we treat relative variations of the temperature, pressure and density amplitudes (see Eqs. (10)-(12)). In 3D model intergranular lanes have a less steep temperature gradient and are cooler in the deeper layers, while granules become cooler in the upper layers of the atmosphere. So, the higher the temperature is the higher the amplitude of its variation has to be. The rapid increase of the intensity amplitude is also present in the 1D MACKKL model atmosphere (see Shchukina \& Khomenko 1999) and in the HSRA atmosphere used by Marmolino \& Stebbins (1989). This disagreement between observations and modeling is possibly due to the effects of wave reflection which were not taken into account. A $5-$ min period wave crosses a certain height in the photosphere (at $\sim 0 \mathrm{~km}$ ) where its period becomes larger than critical at this height and reflects strongly. This should diminish the oscillatory amplitude at the deeper layer.
Let us now discuss the phase shifts of oscillations above granules and intergranular lanes. We computed them for oscillations above the long-living granules (see Sect. 4.2). Figure 10 shows the phase shifts between oscillations of velocity and intensity above these structures. It is striking that the behavior of the intensity and velocity phases is extremely different above granules and lanes (panels a and e). Oscillations of velocity at the continuum level $\delta V_{10 \mathrm{w}}$ lead oscillations at higher layers. The velocity phase shift is much lower (and close to zero) above granules than above intergranular lanes. The interpretation of the phase difference is not straightforward since the dynamics of the local oscillations is very complicated due to contribution of numerous wave trains to the observed phases. According to the series of papers on the dynamics of the solar atmosphere by Deubner \& Fleck (1989, 1990, 1992), Fleck \& Deubner (1989) there are waves observed that propagate energy upward as well as reflected waves carrying energy downward which also contribute to the phase shift. Although the phenomenon of the wave reflection is poorly understood Marmolino et al. (1993) have shown that "in the evanescent region of the $k_{x}-\omega$ diagram between the Lamb waves and the acoustic cut-off frequency, the reflection coefficient is small". Hence, we can expect that the phase of the incident wave should not be affected strongly by the reflected wave in the frequency range under study. The sign of velocity phase obtained in our study suggests that we observe an upward wave propagation. 

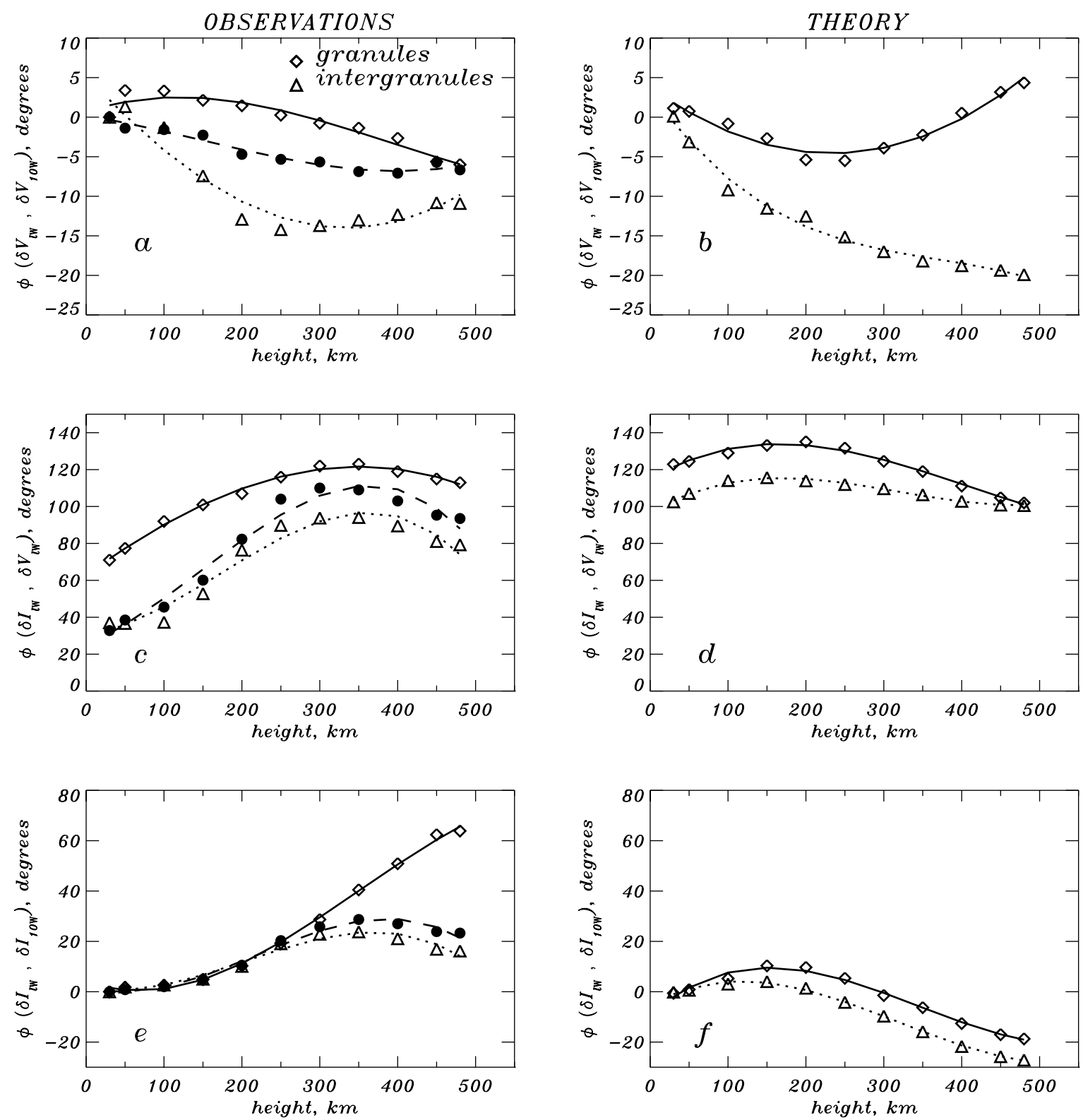

Fig. 10. a) and b) Height dependence of the phase shift between oscillations of velocity at the continuum formation height $l=10$ and at the higher layers $\phi\left(\delta V_{\mathrm{lW}}, \delta V_{10 \mathrm{~W}}\right)$. Positive values mean that the first quantity in the brackets leads and the second lags. In the case of velocity oscillations it means that oscillations at the continuum formation level leads oscillations at higher layers. c) and d) the same case as in panel a) and b) but for the phase shift between intensity and velocity $\phi\left(\delta I_{1 \mathrm{~W}}, \delta V_{\mathrm{lW}}\right)$. e) and $\mathbf{f}$ ) the same but for the phase shift between oscillations of the intensity at the continuum formation level and at the higher layers $\phi\left(\delta I_{\mathrm{lW}}, \delta I_{10 \mathrm{~W}}\right)$. Filled circles in panels a), c) and e) are the values of the corresponding phase shifts calculated without separation into granules and intergranular lanes. All the other notations are the same as in Fig. 9

The absolute values of the velocity phases are rather small so the waves are nearly standing. Note that despite the simplicity of the applied theory it describes rather well the velocity phase behavior.

It is more difficult to interprete the phases of intensity oscillations. The continuum intensity oscillations $\delta I_{10 \mathrm{~W}}$ lag behind oscillations at higher layers both above granules and intergranular lanes. There is no correspondence between computed and observed phase shifts. According to Deubner (1990), "the phase difference observed between intensity fluctuations at different heights in the atmosphere is almost entirely due to the height dependence of the radiation effects action on the $I$ signal". Hence, intensity phases contain information about the physical properties of the media and we can not say anything about the direction of the wave propagation relying on them.

As we see, phases of velocity and intensity differ strongly above granules and above lanes. Their difference, namely the phase shift between intensity and velocity $\phi\left(\delta I_{\mathrm{lW}}, \delta V_{\mathrm{lW}}\right)$, also depends on the granulation contrast. The phase shift $\phi\left(\delta I_{\mathrm{lW}}, \delta V_{\mathrm{lW}}\right)$ is lower above the lanes at 
all heights. This is true both for theory and observations. However, the observed phase shift decreases duwn to 40 degrees in deeper layers. There is no such a decrease in the simulations. As was pointed out by many authors (see for example Marmolino \& Stebbins 1989) within the frame of the used simplified model of wave propagation, the phase shift between intensity and velocity remains at about 90-100 degrees at all heights. Introducing the granular velocity into the model does not improve the situation.

In Figs. 10a, c and e filled circles represent the spatially averaged values of the phase shifts. They were measured at every spatial point and averaged over space without any separation with contrast. These spatially averaged values are located between granular and intergranular values of the phase shifts. It gives a justification that, even if the number of long-living granules is not large, our results are physically meaningful.

As far as we know this study is the first attempt to measure variations of the amplitudes of oscillations above granules and intergranular lanes with height.

We can compare our results of the average granular and intergranular data shown in Figs. 9 and 10 with other studies where the height information was inspected. Our averaged velocity amplitude and phases agree very well with the observational data of Marmolino \& Stebbins (1989) (based on Stebbins \& Goode 1987). In their paper observations of the nonmagnetic Fe I $5434.5 \AA$ line were used. This spectral line forms over all the photosphere as our Fe I $5324 \AA$ line. However, the intensity amplitude behaviour is somewhat different: in particular, we do not observe its amplification with height. Our velocity amplitudes and phase shifts are also consistent with the results of Ruiz Cobo et al. (1997) who applied the inversion techniques method to extract oscillatory properties. The magnitude of the temperature variations given by these authors agrees with the output of the theory of wave propagation we have applied, but its run with height is different. As was pointed out by Ruiz Cobo et al. (1997), the interpretation of the temperature variations following from the inversion is not straightforward because they may be influenced by the variations in the opacity. However, amplitudes of temperature variations calculated by us for the 1D model MACKKL (Maltby et al. 1986) are very similar to those given in Bertello \& Caccin (1990).

Let us remark that before taking into account vertical granular velocities in our calculations, the amplitudes of the velocity oscillations were nearly the same above granules and lanes. Thus, we conclude that velocity oscillations change their behavior mainly because of the interaction of oscillatory and granular velocity fields. Intensity amplitudes remain rather the same in the presence or absence of granular velocity. They are mainly affected by temperature, pressure and density variations.

The disagreement between observed and calculated intensity phases as well as intensity amplitudes may arise both from the simplifications of the wave propagation theory and the utilized 3D model. The analysis of this model performed by Asplund et al. (2000) revealed that it is a realistic representation of solar convection. Consequently, a more sophisticated wave propagation theory may be necessary to explain all the peculiarities of the wave behaviour above granules and intergranular lanes.

\section{Conclusions}

The observations with high spatial $(<0$ '.5) and temporal $(9.3 \mathrm{~s})$ resolution together with theoretical computations allowed us to investigate the local 5-min oscillations above granules and intergranular lanes. In the upper photosphere at a height $\sim 500 \mathrm{~km}$, the amplitudes of intensity oscillations above granules are lower than above lanes. The large-amplitude velocity oscillations occur above both these structures. Velocity oscillations above the lanes appear with the same period through the photosphere, while above granules the period changes with height. The phase shift of velocity oscillations above granules is nearly zero and negative above intergranular lanes. The intensity phase shift is positive and much larger above granules than above the lanes. The difference in oscillations is present throughout the photosphere and increases with height.

Many observational studies have been dedicated to the analysis of peculiarities of the wave behavior above granules and lanes. As was pointed out in Sect. 1, many authors prefer to think that the powerful oscillations occur above the dark intergranular lanes. Our high-resolution observations demonstrate that the situation is not so simple: for example powerful velocity oscillations occur both above granules and lanes but with different period.

There are several possible explanations of the enhancement of the oscillatory amplitude above intergranular lanes. Firstly, oscillations may be generated more efficiently in these structures. Namely, according to hydrodynamical simulations by Stein et al. (1989), Stein \& Nordlund (1991), the location of the source of oscillations should be in the downward plunging convective plumes. Secondly, the solar atmosphere should affect propagating waves acting as a filter which amplifies some oscillations and weakens the others. Our study shows that, without invoking any assumption about wave excitation, oscillations above intergranular lanes appear to have larger amplitudes at height $\sim 500 \mathrm{~km}$ even if they have the same amplitudes at the $H=0$. The dynamics of the solar atmosphere is extremely complicated and hence, many mechanisms may be simultaneously operating there. We do not exclude the possibility of oscillations excited with larger amplitudes in intergranular lanes due to an enhanced turbulence and strong velocity gradients (Nesis et al. 1992; Solanki et al. 1996; Nesis et al. 1997). However, our observations demonstrate that the amplitudes are nearly equal at the deep layers at $H=0$ and the difference between oscillations reaches its maximum at $H \approx 500 \mathrm{~km}$, where the granulation patterns completely disappears.

Finally, we conclude: many features of the peculiar behavior of oscillations above granules and intergranular lanes can be explained within a frame of the simplified 
theory of wave propagation without invoking any excitation mechanisms.

Acknowledgements. Partial support by the Spanish DGICYT under project $91-0530$ is gratefully acknowledged. N.G.S. would like to acknowledge the support of the Spanish Ministry of Education for a sabbatical stay at the IAC. The support provided by C. Briand and H. P. Schilling during the observations is gratefully appreciated. We would like to thank M. Vázquez, J. A. Bonet, J. Trujillo Bueno, M. Collados and their colleagues for support, interest and help in our work. Special thanks to M. Asplund for the kind permission to use 3D model atmosphere for the purposes of our study. We are grateful to A. Kosovichev for carefully reading the manuscript and useful suggestions. We acknowledge support from the Netherlands Organization for Scientific Research (NWO). In this research, we employed in Kiev a computer (CCD system) funded by the NWO.

\section{References}

Alissandrakis, C. E., Dialetis, D., \& Tsiropoula, G. 1987, A\&A, 174,275

Asplund, M., Nordlund, Å., Trampedach, R., Allende Prieto, C., \& Stein, R. F. 2000, A\&A, 359, 729

Asplund, M., Nordlund, A., Trampedach, R., \& Stein, R. F. 1999, A\&A, 346, L17

Auer, L. H., Fabiani Bendicho, P., \& Trujillo Bueno, J. 1994, A\&A, 292, 599

Bertello, L., \& Caccin, B. 1990, A\&A, 231, 509

Brown, T. M. 1991, ApJ, 371, 396

Brown, T. M., Bogdan, T. J., Lites, B. W., \& Thomas, J. H. 1992, ApJ, 394, L65

Delbouille, L., Neven, L., \& Roland, G. 1973, Photometric atlas of the solar spectrum from $\lambda 3000$ to $\lambda 10000$, Institut d'Astrophysique de l'Université de Liège, Liège, Belgium

Deubner, F. L. 1990, in The Solar Photosphere: Structure, Convection and Magnetic Fields, ed. J.-O. Stenflo, Proceedings IAU Symposium 138, Kiev (Kluwer, Dordrecht), 217

Deubner, F.-L., \& Fleck, B. 1989, A\&A, 213, 423

Deubner, F.-L., Fleck, B., Marmolino, C., \& Severino, G. 1990, A\&A, 236, 509

Deubner, F.-L., Fleck, B., Schmitz, F., \& Straus, T. 1992, A\&A, 266, 560

Espagnet, O., Muller, R., Roudier, T., Mein, P., Mein, N., \& Malherbe, J. M. 1996, A\&A, 313, 297

Fleck, B., \& Deubner, F.-L. 1989, A\&A, 224, 245

Goldreich, P., \& Keeley, D. A. 1977, ApJ, 211, 934

Goldreich, P., \& Kumar, P. 1988, ApJ, 326, 462

Goldreich, P., Murray, N., \& Kumar, P. 1994, ApJ, 424, 466

Goode, P. R., Gough, D., \& Kosovichev, A. 1992, ApJ, 387, 707

Gurtovenko, E. A., \& Kostik, R. I. 1989, Fraunhofer Spectrum and the System of Solar oscillator Strengths (Russian Language Edition, Naukova Dumka, Kiev)

Hirzberger, J., Bonet, J. A., Vázquez, M., \& Hanslmeier, A. 1999, ApJ, 515, 441

Hirzberger, J., Vázquez, M., Bonet, J. A., Hanslmeier, A., \& Sobotka, M. 1997, ApJ, 480, 406
Hoekzema, N. M., Brandt, P. N., \& Rutten, R. J. 1998a, A\&A, 333,322

Hoekzema, N. M., \& Rutten, R. J. 1998, A\&A, 329, 725

Hoekzema, N. M., Rutten, R. J., Brandt, P. N., \& Shine, R. A. 1998b, A\&A, 329, 276

Holweger, H., Bard, A., Kock, A., \& Kock, M. 1991, A\&A, 249, 545

Karpinsky, V. N. 1990, in The Solar Photosphere: Structure, Convection and Magnetic Fields, ed. J.-O. Stenflo, Proceedings IAU Symposium 138, Kiev (Kluwer, Dordrecht), 23

Kostik, R., \& Shchukina, N. 1999, Astron. Lett., 25(10), 678

Kostik, R. I. 1974, A\&A, Russian edition, 23, 52

Kumar, P., \& Goldreich, P. 1989, ApJ, 342, 558

Kumar, P., \& Lu, E. 1991, ApJ, 375, L35

Maltby, P., Avrett, E. H., Carlsson, M., et al. 1986, ApJ, 306, 284

Marmolino, C., \& Severino, G. 1991, A\&A, 242, 271

Marmolino, C., Severino, G., Deubner, F.-L., \& Fleck, B. 1993, A\&A, 278, 617

Marmolino, C., \& Stebbins, R. T. 1989, Solar Phys., 124, 23

Ruiz Cobo, B., Rodríguez Hidalgo, I., \& Collados, M. 1997, ApJ, 488, 462

Trujillo Bueno, J., \& Fabiani Bendicho, P. 1995, ApJ, 455, 646 Mihalas, D., \& Mihalas, B. W. 1984, Foundations of Radiation Hydrodynamics (Oxford University Press, Oxford)

Moore, C. E., Minnaert, M. G. J., \& Houtgast, J. 1966, The Solar Spectrum $2935 \AA$ to $8770 \AA$. Second Revision of Rowland's Preliminary Table of Solar Spectrum Wavelengths, NBS Monograph 61, National Bureau of Standards, Washington

Nesis, A., Hammer, R., Hanslmeier, A., et al. 1997, A\&A, 326, 851

Nesis, A., Hanslmeier, A., Hammer, R., et al. 1992, A\&A, 253, 561

Nordlund, Å., \& Stein, R. F. 1990, Comp. Phys. Comm, 59, 119

Noyes, R. W., \& Leighton, R. W. 1963, ApJ, 138, 631

Restaino, S. R., Stebbins, R. T., \& Goode, P. R. 1993, ApJ, 408, L57

Rimmele, T. R., Goode, P. R., Harold, E., \& Stebbins, R. T. 1995, ApJ, 444, L119

Schmieder, B. 1977, Solar Phys., 54, 269

Schröter, E. H., Soltau, D., \& Wiehr, E. 1985, Vistas Astron., 28,519

Shchukina, N., \& Khomenko, E. 1999, in Cool stars, stellar systems and the Sun, 11th, Cambridge workshop, in press

Shchukina, N. G., \& Trujillo Bueno, J. 1997, Solar Phys., 172, 117

Shchukina, N. G., \& Trujillo Bueno, J. 2000, ApJ, accepted

Solanki, S. K., Rüedi, I., Bianda, M., \& Steffen, M. 1996, A\&A, 308,623

Stebbins, R. T., \& Goode, P. R. 1987, Solar Phys., 110, 237

Stein, R. F., \& Nordlund, Å. 1989, ApJ, 342, L95

Stein, R. F., \& Nordlund, A. 1991, ApJ, 342, L95

Stein, R. F., \& Nordlund, Å. 1998, ApJ, 499, 914

Stein, R. F., Nordlund, Å., \& Kuhn, J. R. 1989, in Solar and Stellar Granulation, ed. R. J. Rutten, \& G. Severino (Kluwer Acad. Publ, Dordrecht), 381

Title, A. M., Tarbell, T. D., Topka, K. P., et al. 1989, ApJ, 336, 475 